\title{
Pilotstudie: Propriozeptive Einlegesohlen bei Fußbeschwerden
}

\section{Aktiv stimulierende Einlagen zur Korrektur von Fußdeformitäten und Fehlfunktionen - Vielversprechende Ergebnisse einer Praxis- Pilotstudie \\ Hans Garten}

\section{Fragestellung}

Es gibt eine Vielzahl von Konzepten für die Korrektur von Fußdeformitäten und Fehlfunktionen ebenso wie deren Auswirkungen auf die Gesamtstatik. Grob lassen sich stützende orthopädische Einlagen (passiv) und aktiv stimulierende ohne eine passiv aufrichtende Funktion unterscheiden. Letztere werden auch als "propriozeptive“ oder „sensomotorische“ Einlagen bezeichnet. Die Kriterien zur Definition der Form sind vielfältig und häufig rational schwer nachvollziehbar.

In der Praxis-Pilotstudie sollte die Wirksamkeit eines Sohlensystems, welches aus Sohlenrohlingen und vorfabrizierten Stimulationselementen mit einer Klettverbindung besteht, geprüft werden.

\section{Methodik}

Die Patienten wurden in 3 Gruppen aufgeteilt:

1. Fußbeschwerden (Metatarsalgie, Fasciitis plantaris/Fersensporn, unspezifische Fußschmerzen bei Knick-Senk-Spreizfuß)

2. Haltungsabweichungen mit rezidivierenden Dysfunktionen des Bewegungssystems (Beckendysfunktionen, Kniedysfunktionen)

Abb. 1 Muskeln der Mittelloge an der Fußsohle (rechter Fuß, Ansicht von plantar). Um den Ansatz des M. quadratus plantae am lateralen Rand der Sehne des M. flexor digitorum longus darstellen zu können, wurde der M. flexor digitorum brevis bis auf seinen Ursprung entfernt. Aus: Schünke M, Schulte E, Schumacher U. Prometheus. Lernatlas der Anatomie. Stuttgart: Thieme; 2005. Illustration: Karl Wesker

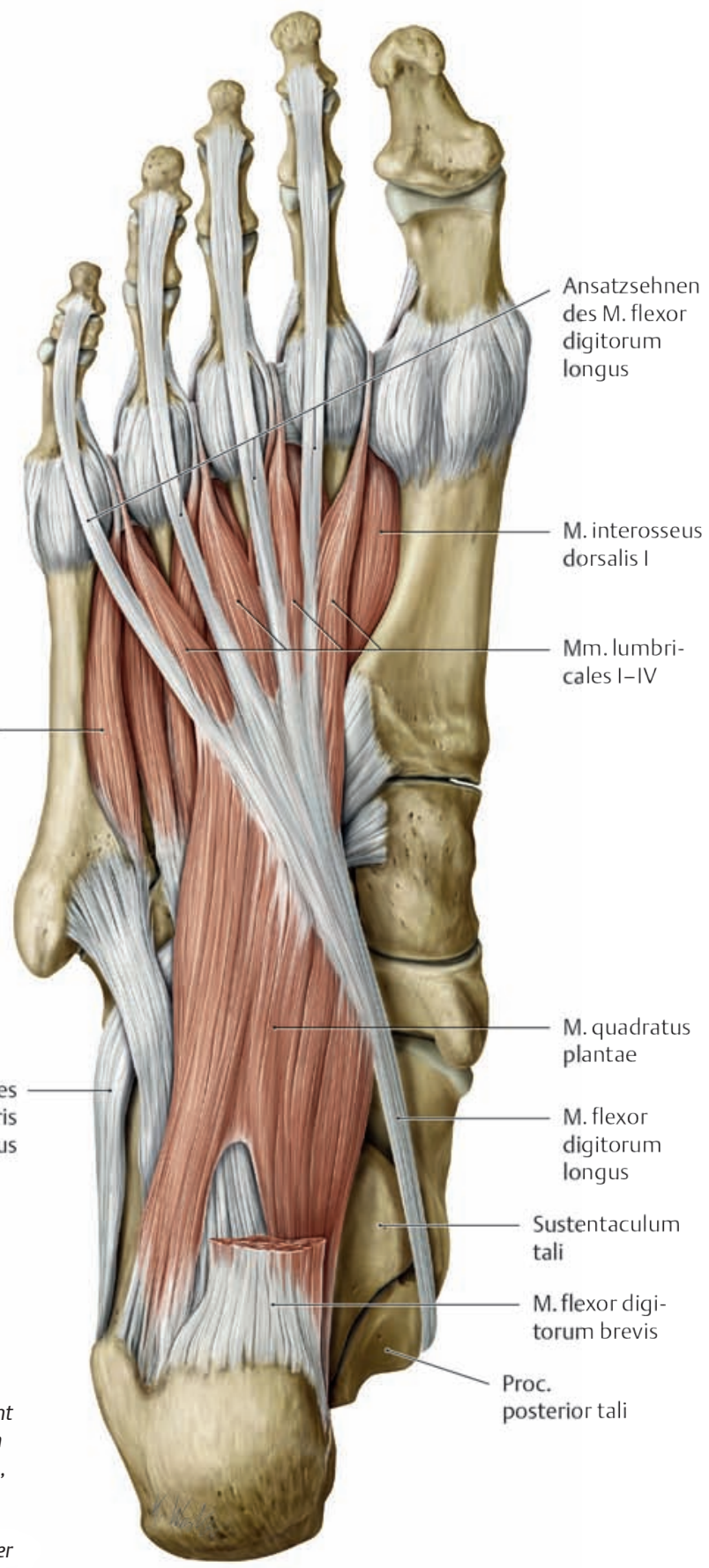




\section{Zusammenfassung}

In der Pilotstudie wurde die Wirkung propriozeptiv wirksamer Einlegesohlen bei den Indikationen Fußbeschwerden (Gruppe 1), Dysfunktionen des Bewegungssystems (Gruppe 2) und neurologische Störungen (Gruppe 3) geprüft. Die Einlegesohlen wurden mit Untersuchungstechniken der Professional Applied Kinesiology in den 3 Indikationsgruppen angepasst.

Ergebnisse waren: Gruppe 1: $(n=15)$ VAS vor Sohlenversorgung Mittelwert 5,13; VAS nach Sohlenversorgung Mittelwert 1,87. Gruppe 2: Knie $(n=7)$, VAS-Mittelwert vor 4,71, nach 2,42; Becken $(n=7)$ VAS-Mittelwert vor 5,14 ; nach 3. Gruppe $3(n=8)$ : Mittelwert Vertrauensellipse [mm2] vor 363,71 nach 166,83 .

In allen Gruppen konnte eine deutliche Symptombesserung auf der visuellen Analogskala sowie in der posturographischen Messung (Vertrauensellipse) erreicht werden.

3. Neurologische Störungen (sensomotorische Neuropathie bei neurodegenerativen und metabolischen Störungen, Parkinson-Syndrom, Schwindel unterschiedlicher Genese)

Nach Kriterien posturologischer Forschung [1-7] und funktionell neurologischen Kriterien wurden unter Sohlenrohlinge stimulierende Elemente platziert und das Ergebnis mittels posturographischen Messungen (Vertrauensellipse) bzw. visueller Analogskala (VAS, Beschwerdeskala zwischen 0 und 10) überprüft.

Die Anfertigung der Sohlen folgte einem festen Diagnoseschema (im Detail dargestellt in [8]):

- Korrektur von muskulären und artikulären Dysfunktionen der unteren Extremität, des Beckens, der Wirbelsäule und des kraniomandibulären Systems

- Ein Belastungschallenge (Gehen, Springen) führte zum Rezidiv von Störungen

- sofort

- nach längerer Belastung bis zum nächsten Patientenkontakt

- Standanalyse auf posturographischer Messplatte (Gruppe 3), dynamischer Fußabdruck auf Blaupause oder Messplatte (Gruppe 1 und 2); in jedem Falle: posturolgischer Spiegelkasten

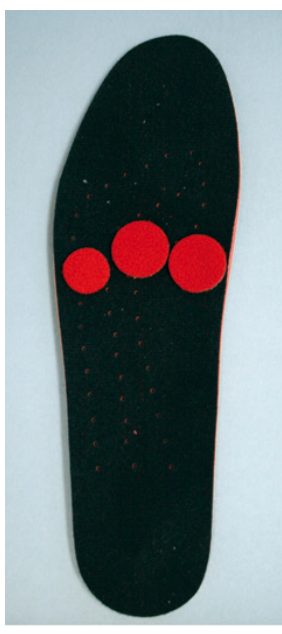

Vorfußpads

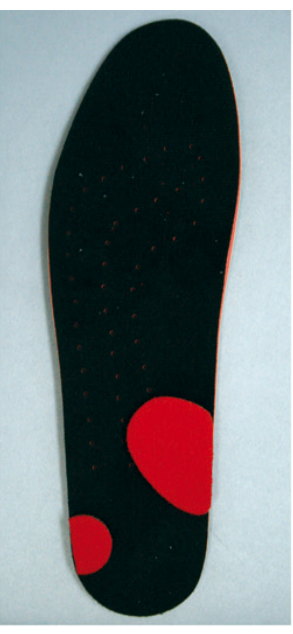

Rückfußpads

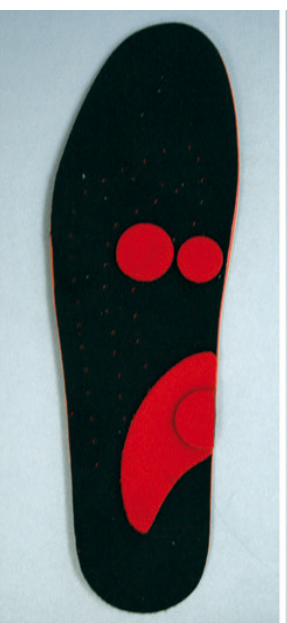

mediale Pads

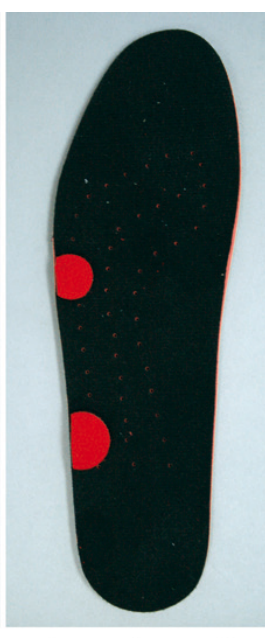

laterale Pads

Abb. 2 Globale Verlagerung des Schwerpunkts erfolgt jeweils weg von den stimulierenden Sensopads.

- Ganganalyse visuell

- aufsteigende Befunde wurden ohne Sohlen oder Einlagen im Stehen erhoben:

- Beckenniveau, Schulterniveau, Okziputniveau, axiale Torsionen

- Sprunggelenksachsen (Pes varus/valgus), Beinachsen (Genu varum/valgum), Rotation der Beine

- Längsgewölbe des Fußes (Pes planus, excavatus), Quergewölbe (Spreizfuß), Hallux valgus, Hammerzehen?

- Rombergversuch neutral und mit Provokationspositionen der HWS

- Manuelle Muskelfunktionsbefunde (M. latissimus dorsi, Mm. rhomboidei, M. serratus ant., oberer Trapezius, M. sternocleidomastoideus, Nackenextensoren). Nackenflexoren und -extensoren [9]

- Therapielokalisationen der Applied Kinesiology des kraniozervikalen und des zervikothorakalen Übergangs sowie des lumbosakralen Übergangs und der SIGs

- Testung der Kaumuskulatur (Kieferheber, Kiefersenker)

- alternierender Cover-Test (zur Dokumentation einer Heterophorie, d.h. latentem Schielen) [10]

- im Sitzen: Ausschlussdiagnostik vestibulärer Störungen: Halmagyi-Headthrust-Test [11]

- im Liegen:

- Definition Cat 1, Cat 2-Beckenläsionen (Ilium ant. bzw. post.) [12]
- Testung der zugehörigen Muskelhyporeaktionen am Becken

- Testung der Sprunggelenksmuskeln

- Die Rohlinge der propriozeptiven Einlegesohlen wurden nach den unten aufgezeigten Regeln probatorisch mit Pelotten versehen.

- Der Patient wurde auf den Sohlen stehend erneut getestet: Eine möglichst große Zahl der erhobenen Befunde sollte durch die Einlegesohlen beseitigt sein.

- Die Position, Form und Größe der Pelotten wurde so lange verändert, bis Letzteres optimal der Fall ist.

\section{Schluss-Challenge}

1. Der Patient ging mit den fertigen Sohlen mindestens einige Schritte (8er-Figur), besser etwa 5 Minuten.

2. Als Schluss-Challenge für alle Systeme galt der Walking-gait-Test: Dort sollte ein vorhersehbares Muskelinhibitionsmuster auftreten [9, 12].

3. Die Schuhe wurden ausgezogen und im Liegen die Befunde am Becken und der Sprunggelenksmuskulatur nachgetestet.

\section{Prinzipien zur Positionierung der Pelotten}

1. Prinzip (postural, Bein- und Körperachsen)

Die Fuß- und Beinachse sowie der Körperschwerpunkt bewegen sich weg vom stimulierenden Element (Abb.2). 


\section{Prinzip (Tonisierung und Inhibition von plantaren Muskeln)}

Flächigere Stimulationselemente unter dem Muskelbauch führen zur Tonisierung des stimulierten Muskels, kleinere Elemente im Bereich des Muskel-Sehnen-Übergangs führen zu Detonisierung.

\section{Ergebnisse}

Die Sohlenversorgung zeigte folgende Ergebnisse:

Gruppe 1: VAS vor Sohlenversorgung Mittelwert 5,13; VAS nach Sohlenversorgung Mittelwert 1,87 (Abb.3).

Gruppe 2: Knie ( $n=7)$, VAS-Mittelwert vor 4,71, nach 2,42 (Abb.4). Becken ( $n=7$ ) VASMittelwert vor 5,14; nach 3 (Abb.5).

Gruppe $3(n=8)$ : Mittelwert Vertrauensellipse [mm2] vor 363,71 nach 166,83; (Abb.6). Unter „Vertrauensellipse“ versteht man die Fläche, die der Posturograph vom realen Schwerpunkt des Patienten aufzeichnet, die durch das Schwanken beim Rombergversuch entsteht.

\section{Schlussfolgerung}

Das präsentierte individualisierte System propriozeptiver Einlegesohlen ist wirksam bei Fußbeschwerden wie Fasciitis plantaris (Fersensporn), Metatarsalgie, Schmerzen bei Hallux valgus und Knick-Senk-Spreizfuß. Ebenso bei Beschwerden des Bewegungssystems: Gonalgien durch statische Störungen und arthrotische Veränderungen, rezidivierenden Beckenstörungen wie Sakroiliakalgelenksdysfunktionen, Beckenverwringungen mit aufsteigenden Folgen im Bereich des Schultergürtels und des kraniomandibulären Systems sowie neurologischen Störungen, insbesondere Gang- und Standunsicherheit bei sensorischer Neuropathie, Parkinsonsyndrom und zerebellärer Ataxie sowie Schwindel unspezifischer Genese.

Es ist kostengünstig und einfach anwendbar: Die Sohle wird nach funktionellen Kriterien direkt vom Anwender hergestellt und ist leicht veränderbar, bis ein optimales Ergebnis erzielt wird.

Interessenkonflikt: Der Autor ist Entwickler der sensomotorischen Einlegesohlen und Inhaber der Firma Restart.

Online zu finden unter

http://dx.doi.org/10.1055/s-0036-1584373

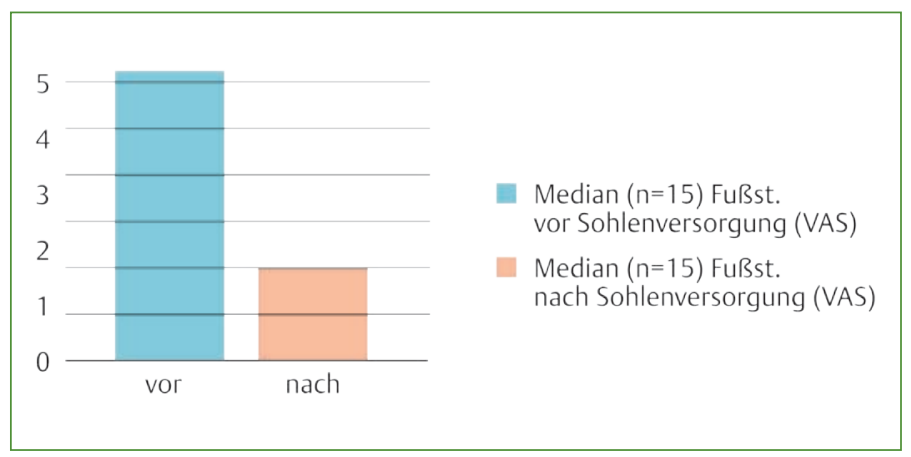

Abb. 3 VAS vor (blau) und nach (rot) Sohlentherapie bei Fußproblemen in Gruppe 1.

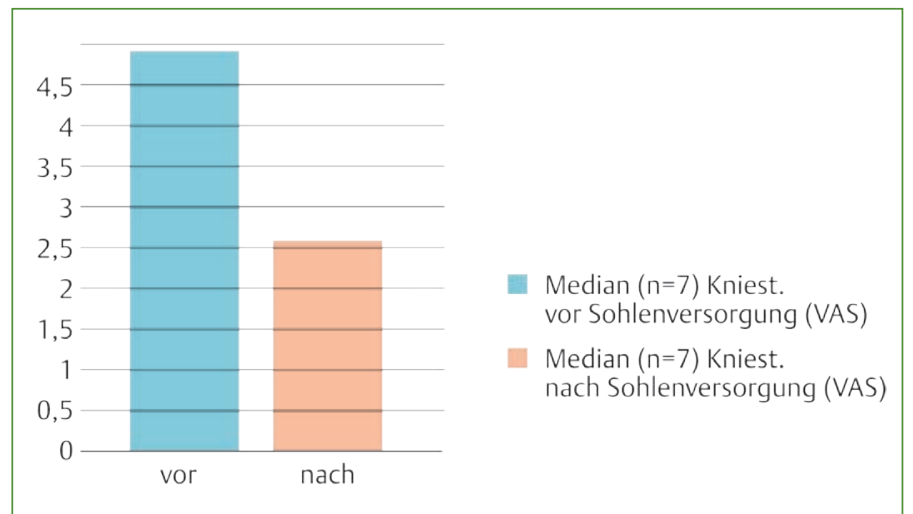

Abb. 4 VAS vor (blau) und nach (rot) Sohlentherapie bei Knieproblemen in Gruppe 2.

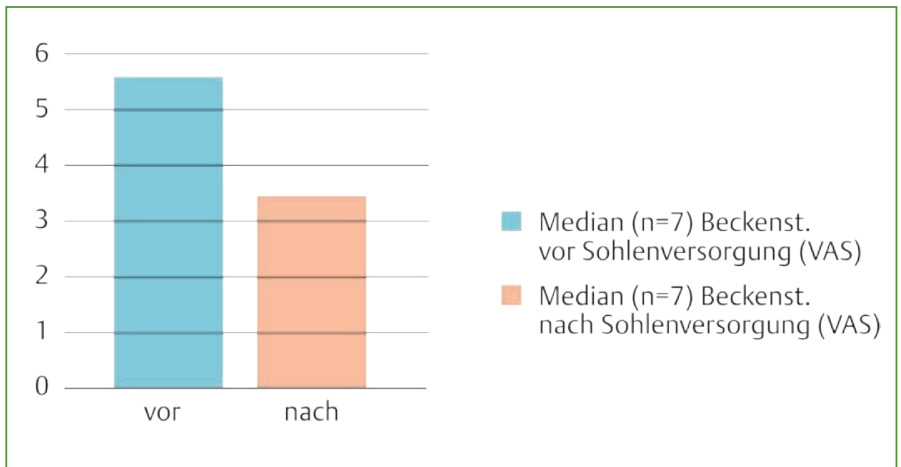

Abb. 5 VAS vor (blau) und nach (rot) Sohlentherapie bei chronischen Störungen des Beckens in Gruppe 2.

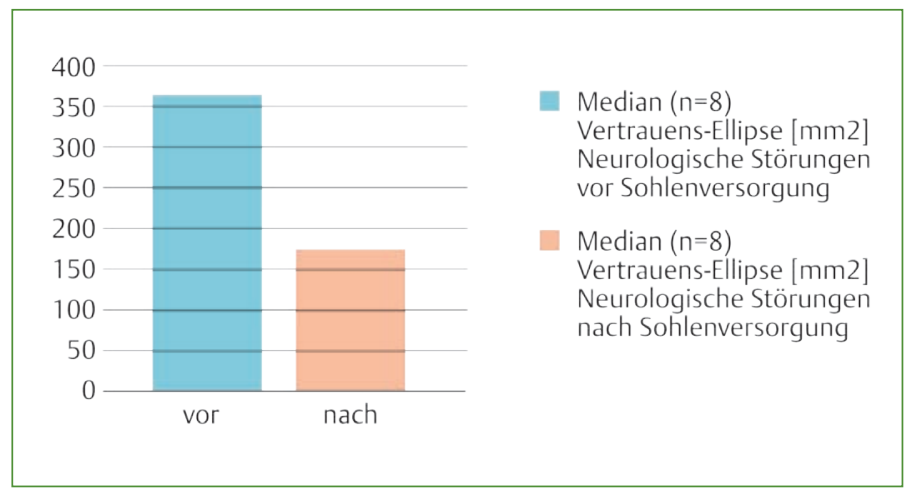

Abb. 6 Vertrauensellipse posturographisch vor (blau) und nach (rot) Sohlentherapie bei neurologischen Störungen in Gruppe 3. 
(C) Der Artikel wurde erstveröffentlicht in: JPAK 2013; 3-6

\section{Literatur}

1 Kavounoudias $\mathrm{A}$ et al. From balance regulation to body orientation: two goals for muscle proprioceptive information processing? Exp Brain Res 1999; 124 (1): 80-88

2 Kavounoudias A, Roll R, Roll JP. The plantar sole is a "dynamometric map" for human balance control. Neuroreport 1998; 9 (14): 3247-3252

3 Kavounoudias A, Roll R, Roll JP. Specific wholebody shifts induced by frequency-modulated vibrations of human plantar soles. Neurosci Lett 1999; 266 (3): 181-184

4 Kavounoudias A, Roll R, Roll JP. Foot sole and ankle muscle inputs contribute jointly to human erect posture regulation. J Physiol 2001; 532 (Pt 3): 869-878

5 Roll JP, Roll R. From Eye to Foot: A Proprioceptive Chain involved in Postural Control. In: Amblard B, Berthoz A, Clarac F, eds. Posture and gait: Development, adaption and modulation. Amsterdam: Elsevier; 1988 $\overline{6}$ Roll JP, Vedel JP, Roll R. Eye, head and skeletal muscle spindle feedback in the elaboration of body references. Prog Brain Res 1989; 80: $113-$ 123; discussion 57-60

$\overline{7}$ Roll R, Kavounoudias A, Roll JP. Cutaneous afferents from human plantar sole contribute to body posture awareness. Neuroreport 2002; 13 (15): 1957-1961

8 Garten H. Praxis der Anwendung propriozeptiver Einlegesohlen. MJAK 2012; 16 (1): 5-13

$\overline{9}$ Garten H. Applied Kinesiology, Muskelfunktion, Dysfunktion und Therapie. 2. Aufl. München: Elsevier; 2012

$\overline{10}$ Leigh RJ, Zee DS. The Neurology of Eye Movements. 3 rd ed. New York: Oxford Press; 1999

$\overline{11}$ Halmagyi Headthrust Test. Im Internet: www. youtube.com

$\overline{12}$ Walther DS. Applied Kinesiology, Synopsis. 2nd ed. Pueblo, Colorado: Systems D.C.; 2000

$\overline{13}$ Putz R, Pabst R, Hrsg. Sobotta Anatomie des Menschen. 22. Aufl. München: Urban \& Fischer; 2007

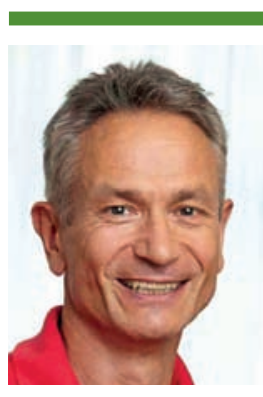

Dr. med. Hans Garten

Nederlingerstr. 35

80638 München

hans.garten@gmx.com 